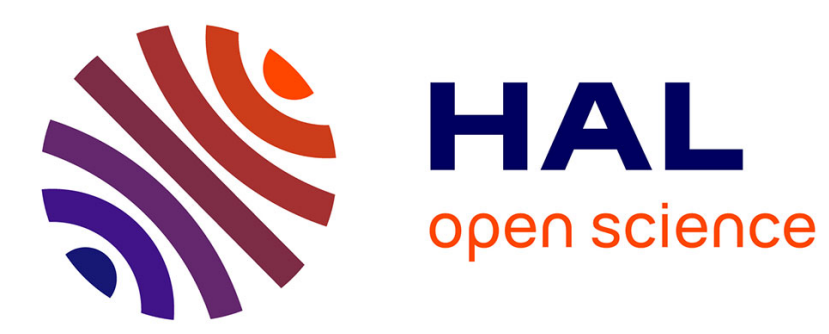

\title{
A Simple Layered RGB BRDF Model
}

Xavier Granier, Wolfgang Heidrich

\section{- To cite this version:}

Xavier Granier, Wolfgang Heidrich. A Simple Layered RGB BRDF Model. Graphical Models, 2003, 65 (4), pp.171-184. 10.1016/S1524-0703(03)00042-0 . hal-00308260v2

\section{HAL Id: hal-00308260 https://hal.science/hal-00308260v2}

Submitted on 27 Oct 2013

HAL is a multi-disciplinary open access archive for the deposit and dissemination of scientific research documents, whether they are published or not. The documents may come from teaching and research institutions in France or abroad, or from public or private research centers.
L'archive ouverte pluridisciplinaire HAL, est destinée au dépôt et à la diffusion de documents scientifiques de niveau recherche, publiés ou non, émanant des établissements d'enseignement et de recherche français ou étrangers, des laboratoires publics ou privés. 


\title{
A Simple Layered RGB BRDF Model
}

\author{
Xavier Granier ${ }^{1}$ and Wolfgang Heidrich \\ IMAGER,University of British Columbia, Department of Computer Science \\ 201-2366 Main Mall Vancouver, B.C. V6T 1Z4, Canada
}

\begin{abstract}
Many natural objects, and in particular layered materials, have non-linear reflection behavior along the wavelengths. An accurate representation of phenomena such as interference and color dispersion generally requires a fine spectral representation of light instead of the commonly used RGB components. In this article, we introduce and experiment with a general approach to create similar and convincing effects, with a simple RGB BRDF model. We present a model for both specular and a diffuse reflection, and implement them into software and hardware accelerated rendering process.
\end{abstract}

Key words: BRDF, reflection model, interference, spectral effects, color dispersion

\section{Introduction}

The bi-directional reflectance distribution function (BRDF) has proven its efficiency to describe complex light interactions with surfaces. A large range of material appearances can be represented by this local approach, as it can be noticed in the high quality of the today's computer generated images. BRDF models have played an essential role in creating realistic illumination of the virtual worlds.

In order to simulate effects such as old metallic objects or those with a thin transparent layer, with a wavelength-dependent refraction index, it is necessary to make the BRDFs dependent on the wavelength, thereby adding an

\footnotetext{
Email address: $\{$ xgranier,heidrich\}@cs.ubc.ca (Xavier Granier). $U R L$ : www.cs.ubc.ca/ ${ }^{\sim}$ xgranier, heidrich $\}$ (Xavier Granier).

1 PIMS Post-doctoral Fellow(http://www.pims.math.ca)
} 
additional dimension. Most previous work in this area [Stam (1999); Hirayama et al. (2000a); Sun et al. (2000); Icart and Arques (2000)], is based on a fine spectral representation of the light. That work is focused on accuracy for a physical simulation of the light reflection.

Because RGB is the most commonly used light representation in computer graphic, and since the final display process uses the same encoding, we want to explore the possibility of creating such effects using only these three components. With this common restriction, the resulting models can be merged more easily in the current image production process.

Thus, our main goal is to create a realistic and a convincing model, even if it is not necessarily an accurate physical model. We will mainly focus on the simplicity, and on the low number of parameters.

\subsection{Overview}

In this paper, we introduce a new BRDF model, based on the commonly used RGB representation of light. This model allows computing some prominent wavelength-dependent effects.

We begin by reviewing the existing work on the topic, which is mainly focused on creating accurate models for such effects, in general based on a fine spectral representation. Then, we will present our general approach to create interference and color decomposition with a simple RGB basis. With this approach, we will describe two possible implementations, one for the specular case, one for the diffuse case. Then we will show, by some results, the possible range of appearances that this kind of approach allows to compute, both for software and hardware rendering.

\section{Previous Work}

A lot of work has been done during the past thirty years in order to create BRDF models that have a convincing behavior, from the well-known Phong model [Phong (1975)] and its adaptation by Lafortune et al. [Lafortune and Willems (1994)] to make it more physically plausible or to extend if in a more general way [Lafortune et al. (1997)]. Many complex representations have also been developed to simulate the influence of the underlying geometry (micro-facet model [Torrance and Sparrow (1967); Ashikhmin et al. (2000)]), or the subsurface scattering [Hanrahan and Krueger (1993)], or to obtain an accurate representation by a real physical model [He et al. (1991)]. This last 
one accurately models wavelength behavior, but can't represent phenomena like interference and color dispersion.

To allow for fast computation and an easy integration in global illumination problem (for an easy stochastic sampling), Ward [Ward (1991)] and Schlick [Schlick (1994)] have introduced simpler models, between empirical and physical. Together with the Phong model and its variants, these are today the most commonly used due to their efficiency and their simplicity.

But despite the complexity of, for example, the model by He et al. [He et al. (1991)], none of these representations can simulate a number of non-linear wavelength effects. One of these effects is the diffraction due to a non-planar surface when the size of underlying micro-geometry reaches the wavelength size, like those we can see on a CD-ROM surface for example. Stam [Stam (1999)] has introduced a general reflection model for these effects, and Sun et al. [Sun et al. (2000)] a model restricted to the CD-ROM simulation. All these methods require multiple wavelength samples to have convincing results and cannot deal with the phenomena due to a layered surface.

Icart et al. [Icart and Arqus (1999); Icart and Arques (2000)] have introduced physically based models for multiple layered surfaces, for both layers with parallel boundaries [Icart and Arqus (1999)] and those with uncorrelated one [Icart and Arques (2000)]. These models are based on solid physical assumptions, by are quite complex and require a fine spectral representation of the light. Hirayama et al [Hirayama et al. (1999, 2000b, 2001)] provide also an accurate model for materials with parallel layers and an approximation [Hirayama et al. (2000a)] for rough surfaces. Their model is based on recursive traversal of all the layers and require also this fine discretization of the wavelength.

Since our goal is to provide a simpler model that can compute similar wavelength effects for layered materials, with the RGB base for the color representation, all these previous approaches are too complex for our purpose and require too many wavelength samples. We will focus on the creation of a heuristic model that allow computing convincing appearance, and on the reduction of the complexity of the previous solutions.

\section{General Presentation}

In this section, we present our approximations and the general form of our BRDF model. The detailed implementations will be described in the following sections. 


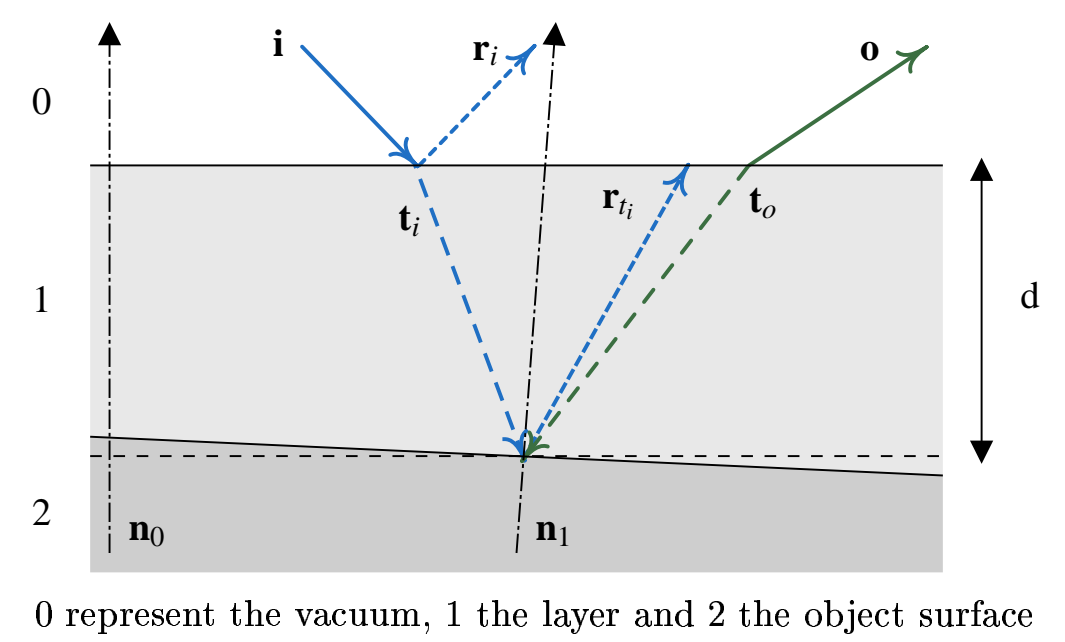

Fig. 1. Local Layer geometry

\subsection{Wavelength Effects}

We can determine two wavelength effects due to the propagation in a layer. The first one is the phase change between the light directly reflected and the part that has traversed the layer. The second is a local prism configuration that can create some color dispersion if the refraction index is varying with the wavelength. In our BRDF model, we want to integrate these two effects.

The phase difference influences the interference between the directly reflected energy and the energy due to the travel through the layer. The two energies can be added or subtracted. The size of the layer is the main influence for the phase change [Born and Wolf (1975)].

The color dispersion is a well-known effect of the prism, for a material with a non-constant refraction index. This effect can also occur in a layered material if the two boundaries of the layer are not parallel. This configuration creates locally the same geometry than a prism.

\subsection{Local Configuration}

To integrate these two effects, we will use the layer configuration described in Fig. 1. The material is composed of a thin layer with uncorrelated boundaries. We make also the classical assumption, that the layer size is small enough to neglect the distance between the directly reflected ray and the ray resulting from propagation through the layer. Then, we suppose that locally, the layer size $d$ is constant and the normal of the two boundaries (between vacuum and layer - from 0 to 1 - and between the layer and the object - from 1 to 2) are

uncorrelated. When the two normals are not aligned, this creates a local prism 
configuration.

Along the surface, we can vary the size of the layer to create different interference effects. We can change also the relative position of the two normals. The normal variation can be correlated to the $d$ variation, then we have only one parameter to make local change. These two parameters can be also independent for, as an example, creating color decomposition with a constant-thickness layer. This independence will increase the range of possible reflection effects.

Note that, in such configuration, the multiple reflections in the layer are quickly absorbed [Hirayama et al. (2000a)]. We will thus use only the reflection term.

\subsection{Phase Computation}

The phase change for a wave after a travel trough a layer with two perfectly parallel boundary surfaces is commonly expressed as

$$
\Phi=2 \pi \frac{r_{1}(\lambda) d}{\lambda}<\mathbf{t}_{i},-\mathbf{n}_{0}>
$$

where $\langle\cdot, \cdot\rangle$ is the dot product, $\lambda$ is the wavelength, and $r_{1}(\lambda)$ the refraction index of the layer [Born and Wolf (1975)]. In this formula $2 d<\mathbf{t}_{i},-\mathbf{n}_{0}>$ represents the distance covered by the ray in the layer along the main axes (i.e. $\mathbf{n}_{0}$ ). This expression is only valid for the mirror direction of the incoming light (i.e. $\mathbf{r}_{i}$ ).

For our case, we need to consider every possible outgoing direction. We can then express more generally the distance covered by the ray by

$$
d\left(<\mathbf{t}_{i},-\mathbf{n}_{0}>+<\mathbf{t}_{o},-\mathbf{n}_{0}>\right)
$$

where $\mathbf{t}_{0}$ depends on the normal $\mathbf{n}_{1}$ of the lower boundary surface. Eq. 1 is now only valid for the special case where the outgoing direction o correspond to the mirror one. The more general phase change can be expressed as

$$
\Phi(\mathbf{i}, \mathbf{o}, \lambda)=\pi \frac{r_{1}(\lambda) d}{\lambda}<\mathbf{t}_{i}+\mathbf{t}_{o},-\mathbf{n}_{0}>
$$

As a first approximation, we will chose a representative wavelength for each color component (645 nm -R, $525 \mathrm{~nm}-\mathrm{G}, 445 \mathrm{~nm}-\mathrm{B}$ as an example) for the estimation of this equation and the followings. 


\subsection{Resulting Energy}

Once we know the phase change, we can compute the resulting energy due to interference between the directly reflected energy $I_{r}$ and the transmitted energy $I_{t}$. It is commonly [Born and Wolf (1975)] expressed as

$$
I_{r}+I_{t}+2 \cos (2 \Phi) \sqrt{I_{r} I_{t}}
$$

We choose then as a general representation of our BRDF models:

$$
\rho(\mathbf{i}, \mathbf{o}, \lambda)=R(\mathbf{i}, \mathbf{o}, \lambda)+T(\mathbf{i}, \mathbf{o}, \lambda)+2 \cos (2 \Phi(\mathbf{i}, \mathbf{o}, \lambda)) \sqrt{R(\mathbf{i}, \mathbf{o}, \lambda) T(\mathbf{i}, \mathbf{o}, \lambda)}
$$

In this equation, $R$ represents a BRDF for the direct reflection and $T$ a BRDF for the transmission part.

We have to be careful when we choose the two functions $R$ and $T$, as we want to be able to compute a convincing solution with only three components. If we choose Dirac-based functions, we will generate, in the best case, the three distinct RGB colors for the three different wavelengths. To allow a smooth transition between the colors, we use a lobe representation, similar to the Phong models [Phong (1975)], to simulate the wavelength dependency of the material.

\section{Phong-like Model}

As said in the previous section, using Phong lobe is the easiest way to create smooth transitions between wavelengths. We will use two independent Phong models, one for the reflection, one for the transmission. This allows us to represent the effects of two uncorrelated and rough boundaries of the layer.

Note that in the following description, we will point out each time that a parameter is wavelength-dependent. By default, a parameter is independent of wavelength.

Note also that we will use the assumption of an increasing refraction index from outside (0) to the inside (2). With this condition, any ray can be refracted. This assumption can be easily removed, by adding a test on the maximum refracted angle. 


\subsection{Reflected Part}

The reflected part $R(\mathbf{i}, \mathbf{o}, \lambda)$ is a Phong reflection weighted by the amount of energy that is directly reflected. It can be then as

$$
R(\mathbf{i}, \mathbf{o}, \lambda)=\mathcal{F}(-\mathbf{i}, \lambda)<\mathbf{r}_{i}, \mathbf{o}>^{e_{0}}
$$

where $\mathcal{F}(-\mathbf{i})$ is the Fresnel term. For efficiency purposes, we chose the Schlick approximation [Schlick (1994)]:

$$
\mathcal{F}(\mathbf{i}, \lambda)=f(\lambda)+(1-f(\lambda))\left(1-<\mathbf{i}, \mathbf{n}_{0}>\right)^{5}
$$

where $f(\lambda)$ is the Fresnel coefficient (i.e. Fresnel term at normal direction):

$$
f(\lambda)=\left(\frac{r_{0}-r_{1}(\lambda)}{r_{0}+r_{1}(\lambda)}\right)^{2}
$$

In this equation $r_{0}$ is the refraction index of air (or vacuum) and $r_{1}(\lambda)$ is the refraction index for the layer for the current wavelength. Considering that $r_{0} \simeq 1$ this reflection is fully determined by only 4 parameters, the RGB refraction indices, and the Phong exponent.

\subsection{Transmitted Part}

We choose the same representation for the transmitted part $\mathcal{T}(\mathbf{v}, \lambda)$. As the sum of the reflected and the transmitted energy is always 1 , the transmission term can be simply obtained by $\mathcal{T}=1-\mathcal{F}$, or:

$$
\mathcal{T}(\mathbf{v}, \lambda)=(1-f(\lambda))\left(1-(1+<\mathbf{v}, \mathbf{n}>)^{5}\right)
$$

The transmitted part can be then written as

$$
T(\mathbf{i}, \mathbf{o}, \lambda)=(1-\mathcal{F}(\mathbf{i}, \lambda)) \mathcal{T}\left(\mathbf{t}_{o}, \lambda\right)<\mathbf{r}_{t i},-\mathbf{t}_{o}>^{e_{1}}
$$

Note that this function has a zero value for the three following conditions:

$$
<\mathbf{t}_{i}, \mathbf{n}_{1}>\geq 0 \vee<\mathbf{t}_{o}, \mathbf{n}_{1}>\geq 0 \vee<\mathbf{r}_{t i}, \mathbf{t}_{o}>\geq 0
$$

They correspond to the case where there is no possible reflection in the layer, due to the local configuration. When the boundaries of the layer are parallels, these three conditions are always false. 
Like for the reflected part, the transmitted part is fully determined by only 4 parameters, the RGB refraction indices, and the Phong exponent.

\subsection{Discussion of the Parameters}

There are two parameters do determine the local configuration and five parameters to describe the BRDF behavior.

The two parameters for the local configuration are the distance, and the normal variation between the two boundaries. The distance influences the color of the reflection, a low one will create in general a red color. If we increase it, blue and green color will appear. The normal variation controls the creation of the color dispersion (rainbow-effect). The greater is normal variation, the larger is the dispersion.

The color decomposition can appear only if at least two of the three refraction indices are different. In general, the refraction index is decreasing with increasing wavelength (i.e. the blue index would be higher than the green one, which would in turn be higher than the red one).

Thus, the two Phong exponents will control the roughness of the two boundaries of the layer, and the interpolation between the different colors. Low exponents will result in a blurry color dispersion, and a too large value will not create the smooth transition that we are expecting.

\section{Diffuse Reflection}

In general, any king of coating on a surface will not only impact the appearance of the specular reflection, but also that of the diffuse component. As we have for the specular part, we want to investigate if we can create a diffuse model on which the layer has an influence. For this case, only the thickness of the layer has an effect on the resulting color, since color dispersion is a purely directional effect.

A diffuse approximation of the Phong-like model can be obtained by averaging it along the incident and reflected directions. But the resulting integration has no simple solution. However, due to the linearity of the integration, it will result in an equation keeping some similarities with the original one Eq. 5:

$$
\rho_{d}=\bar{R}+\bar{T}+2 \overline{\cos (2 \Phi) \sqrt{R T}}
$$


where ${ }^{-}$represents the averaging operator. The resulting diffuse coefficient is still the sum of the reflected part with the transmitted part, and with the interference part. We approximate the diffuse reflection with the same model:

$$
\rho_{d}(\lambda)=R_{d}(\lambda)+T_{d}(\lambda)+2 \cos (2 \Phi(\lambda)) \sqrt{R_{d}(\lambda) T_{d}(\lambda)}
$$

where $R_{d}$ is the diffuse term for the reflected part, $T_{d}$ is diffuse term for the refracted part, and $\Phi$ is an average phase change.

To define $R_{d}$, we simply compute the average value of the reflected part in the Phong-like model (Eq. 6), when the Phong exponent in zero $e_{0}=0$. Thus, we obtain:

$$
R_{d}(\lambda)=\frac{1}{21} \frac{-38 r_{0} r_{1}(\lambda)+21\left(r_{1}(\lambda)^{2}+r_{0}^{2}\right)}{\left(r_{1}(\lambda)+r_{0}\right)^{2}}
$$

Considering that there is no absorption in the layer, we can define $T_{d}=1-R_{d}$, i.e.:

$$
T_{d}(\lambda)=\frac{80}{21} \frac{r_{0} r_{1}(\lambda)}{\left(r_{1}(\lambda)+r_{0}\right)^{2}}
$$

To compute an equivalent phase change between the diffuse reflected and the transmitted part, we can use the previous expression of the phase change (Eq. 3) at the normal direction. In fact, the average refracted cosine (i.e. the average value of $\left\langle\mathbf{t}_{i},-\mathbf{n}_{0}\right\rangle$ ) is:

$$
\frac{1}{4} \frac{\ln \left(\frac{r_{1}(\lambda)+r_{0}}{r_{1}(\lambda)-r_{0}}\right)\left(r_{1}(\lambda)^{2}-r_{0}^{2}\right)+2 r_{1}(\lambda) r_{0}}{r_{1}(\lambda) r_{0}}
$$

When $r_{0} \simeq 1$, this value quickly converges to 1 when $r_{1}(\lambda)$ is increasing (for $r_{1}=1.5$, like glass material, this expression is equal to .85).

Then, we choose as the equivalent phase change the following value

$$
\Phi(\lambda)=2 \pi \frac{r_{1}(\lambda) d}{\lambda}
$$

and the diffuse coefficient becomes

$$
\rho_{d}(\lambda)=1+2 \cos \left(4 \pi \frac{r_{1}(\lambda) d}{\lambda}\right) \sqrt{T_{d}(\lambda)\left(1-T_{d}(\lambda)\right)} .
$$

By combining the diffuse reflection and the specular reflection, we can create a complete model of a layered surface. 


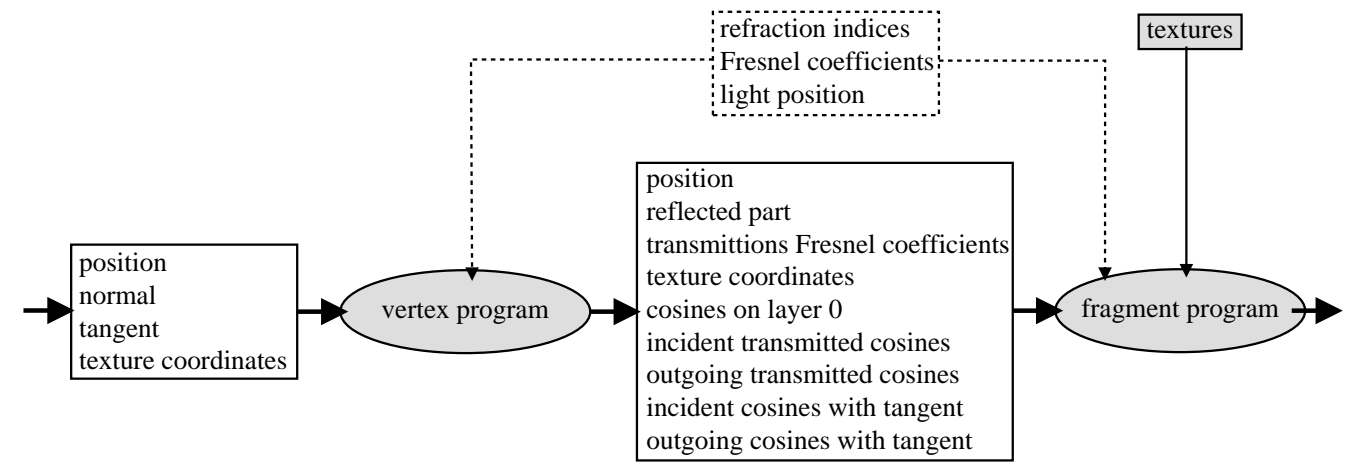

Fig. 2. Hardware implementation diagram

\section{Hardware implementation}

In this section, we describe how an hardware implementation can be done. If the diffuse part can be pre-computed as a texture, the Phong-like model can be implemented using the programmability and the floating point precision available on recent graphic hardware, such as the ATI Radeon 9700 chip. This implementation is based on four components: two textures $T_{\Phi}$ and $T_{d}$, a vertex program, and a fragment program. We use the GL_ARB_vertex_program and $G L_{-} A R B_{-}$fragment_program OpenGL extensions for the programs, and GL_ATI_texture_float for storing the textures with a floating point precision.

As described in the diagram (cf. Fig. 2), we are using the vertex program part to perform all the computations that do not require the layer thickness and the normal variation. We then compute the reflected part, the Schlick approximation of the Fresnel terms for the incoming and outgoing directions, and all the cosines on the external boundaries of the layer (incoming $\langle\mathbf{i}, \mathbf{n}\rangle$, outgoing $\langle\mathbf{o}, \mathbf{n}\rangle$, incoming-outgoing $\langle\mathbf{i}, \mathbf{o}\rangle$, refracted $\left\langle\mathbf{t}_{i}(\lambda), \mathbf{n}\right\rangle, \ldots$ ).

The two textures provide the fragment program with the layer thickness information: the normal variation and the phase change. The first one, a onedimensional RGB texture $T_{\Phi}$, stores the cosine of the phase change for each of the color components (i.e. $2 \cos (2 \Phi(\mathbf{i}, \mathbf{o}, \lambda))$ in Eq. 5):

$$
T_{\Phi}(s)=\left[2 \cos \left(2 \pi \frac{2 d_{\max } r_{1}(\lambda)}{\lambda} s\right), \lambda=R, G, B\right]
$$

where $d_{\max }$ is the maximum thickness of the layer, and $s \in[0,1]$ is the texture coordinate. This coordinate is evaluated for each color component in the fragment program $\left(s=d\left(<\mathbf{t}_{i},-\mathbf{n}_{0}>+<\mathbf{t}_{o},-\mathbf{n}_{0}>\right) /\left(2 d_{\max }\right)\right)$.

The second one, a two-dimensional RGBA texture $T_{d}$, is the equivalent of a classical Bump-Map texture and stores the normalized layer thickness $\left(d /\left(2 d_{\max }\right)\right.$, 


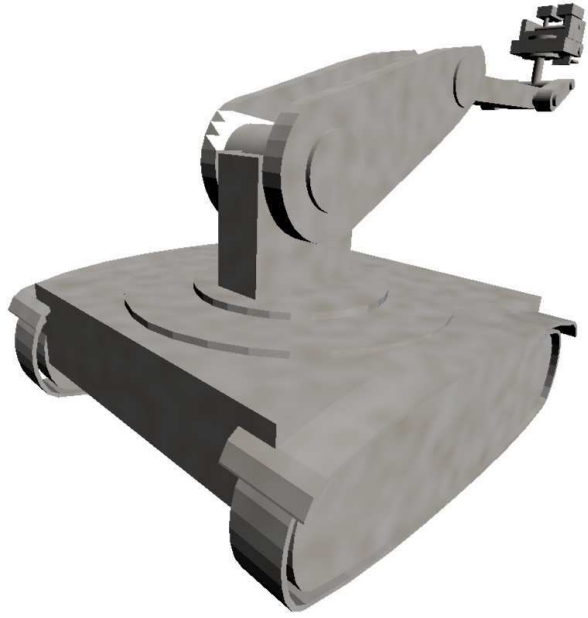

$1-30 \mathrm{~nm}$

refraction index 1.5

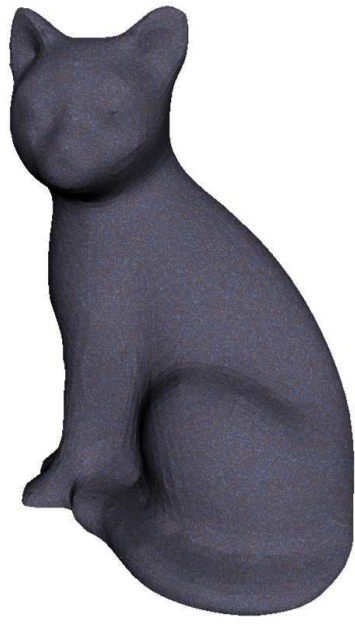

$1-210 \mathrm{~nm}$

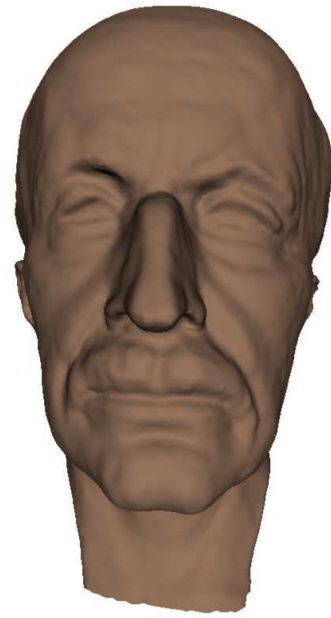

$67-73 \mathrm{~nm}$

1.4

Fig. 3. Diffuse part

the variations on the tangent plane $(\delta d / \delta x$ and $\delta d / \delta y)$ and a normalization factor $\left(\sqrt{1+(\delta d / \delta x)^{2}+(\delta d / \delta y)^{2}}\right)$, corresponding to the cosine between the two normals. The first value will allow us to compute the texture coordinate for the phase texture $T_{\Phi}$; the others are needed to compute the cosines against the normal $\mathbf{n}_{1}$ inside the layer.

In the fragment program, we first compute the transmitted part, using the layer thickness and the normal variation, present in $T_{d}$, and the cosines, that the vertex program provide. With the transmitted cosines computed in the vertex program and the normalize thickness, the texture coordinate for each color component is then evaluated to access to the phase information in $T_{\Phi}$. Then the transmitted part is combined with the reflected part (provided also by the vertex program) as in Eq. 5 .

\section{Results}

In this section, we will present some images from our implementation of the general RGB BRDF model. These results will show the possible range of effects that such an approach can provide. We performed some tests for both the diffuse reflection and the Phong model. The images in Fig. 3 to Fig. 6 are computed using a ray tracing approach, and the images in Fig. 7, using an hardware implementation. 


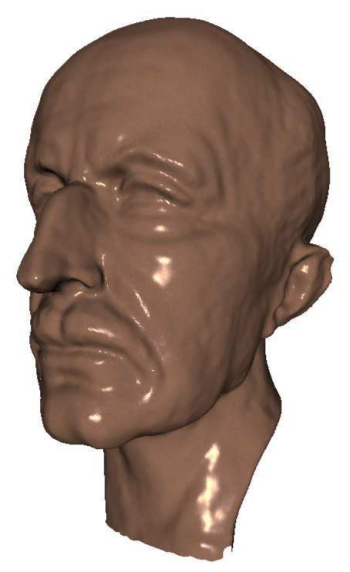

parallel boundaries

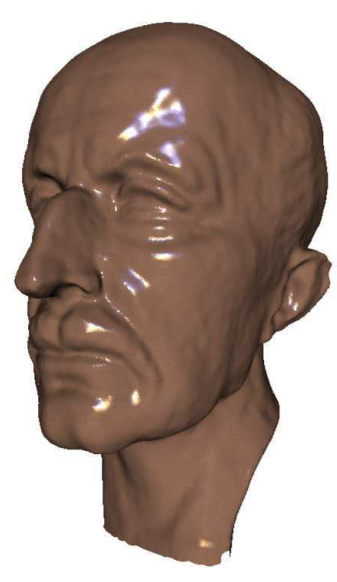

constant deviation

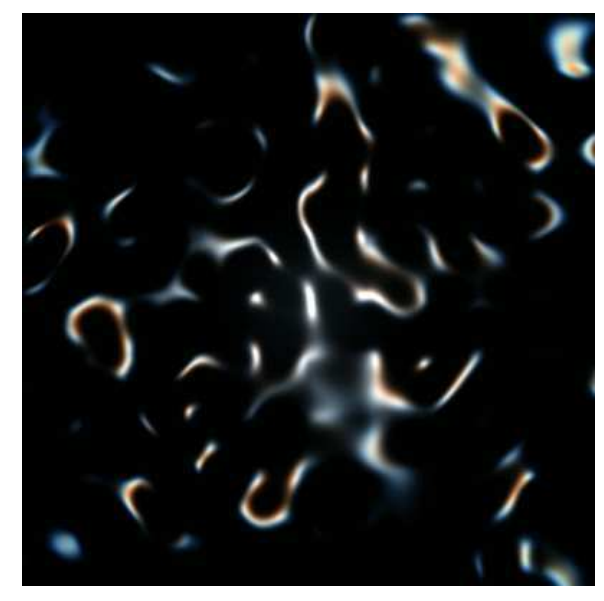

Phong model only

Fig. 4. Normal variation

Fig. 5. Normal/Height correlated

\subsection{Variation of the Layer Thickness}

We use Perlin noise [Perlin (1985)] to compute the variation of the layer size. Then, we can control the color variation by adjusting the range of the layer thickness. The more visible effects appear for diffuse model, as shown in Fig. 3 and Fig. 6.

As explained in the section 4.3, a low layer size will result in general in a red color, and a higher value in blue or green values, has you can see in Fig. 6 .

With only the thickness variation, we can an create the appearance of oldmetal (Fig. 3-left), clay (Fig. 3-right), or some more colored effects (Fig. 3center).

\subsection{Normal Variation}

By introducing a difference between the normal orientation of the two layer boundaries, we create both deviation of the reflected direction, (Fig. $4^{2}$ ), but also a color dispersion when the RGB refraction indices are different (Fig. 4 and Fig. 6). In these examples, the difference between the two normals is constant over the surfaces.

$\overline{2}$ for this figure, the parameters are: Phong exponents $e 0=90, e 1=170$, refraction indices $(1.4,1.45,1.6)$, thickness range 66 to $76 \mathrm{~nm}$, Phong Vs Diffuse: 1-0.7 


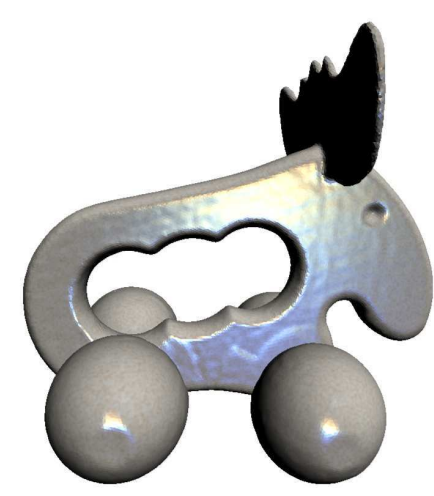

$1-30 \mathrm{~nm}$

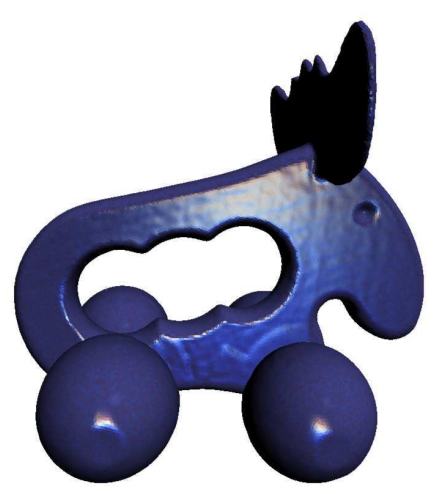

79-106 $\mathrm{nm}$

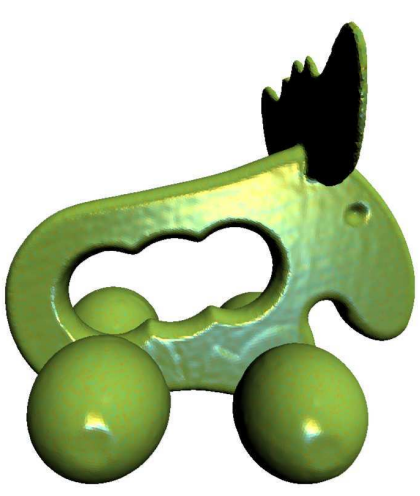

$146-200 \mathrm{~nm}$

For these examples, the RGB refraction indices are respectively 1.5, 1.6 and 1.8, the constant normal deviation is $14^{\circ}$, the Phong exponents are $e 0=e 1=130$ and the weight of the Specular part and the diffuse part are respectively 1 and 0.5.

Fig. 6. Combining specular and diffuse part

\subsection{Combining Both Effects}

We can combine also the normal deviation and the thickness variation independently to create more effects. The thickness will control the base color, and the normal deviation the color decomposition (Fig. 4 and Fig. 6).

But the normal deviation can be also correlated to the variation of the layer thickness (Fig. 5), as for bump-mapping [Blinn (1978)]. This allows creating more effects, like those visible when there is a thin layer of oil on a surface.

\subsection{Hardware Implementation}

We used the hardware implementation of the Phong-like model to produce the image of the figure Fig. 7 . The simulation run at 33 frames per second ${ }^{3}$, for this cat model composed of 11,000 triangles. In this case, the normal variation is correlated with the layer thickness variation. We use a size of 256 for the phase texture $T_{\Phi}$ and $256 \times 256$ for the layer thickness texture $T_{d}$ which has been generated using Perlin's noise. The differences in color between the images correspond only to the change of layer thickness range. You can see also the subtle color variations, that create a quite complex shading.

$\overline{3}$ on a workstation with ATI Radeon 9700 Pro, Intel Pentium III 1 GHz, running GNU/Linux 


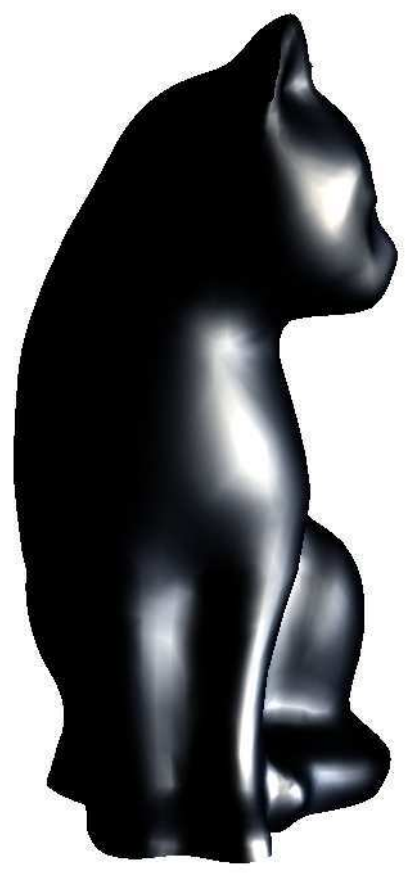

$1-30 \mathrm{~nm}$

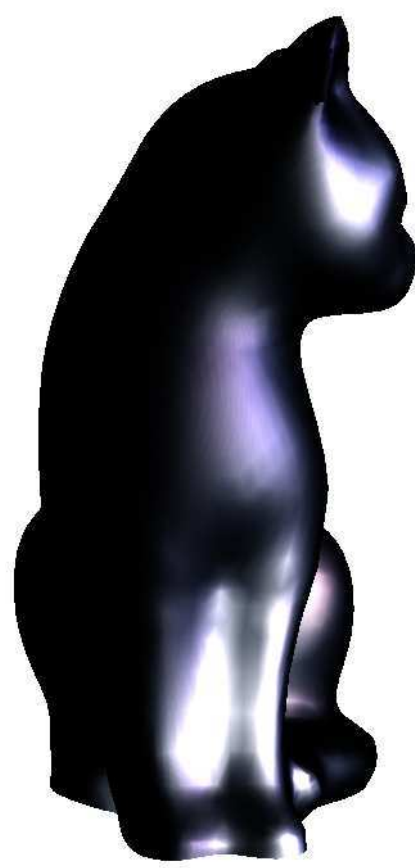

$1-100 \mathrm{~nm}$

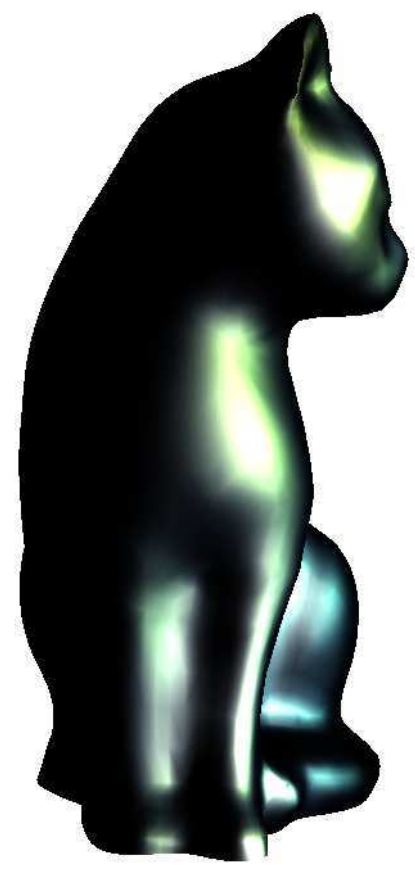

$1-200 \mathrm{~nm}$

Phong exponents: $e_{0}=e_{1}=10 \mathrm{RGB}$ refraction indices: $1.45,1.5,1.6$

Fig. 7. Hardware implementations

\section{Conclusion}

In this paper, we have presented a simple and a general approach to integrate wavelength dependent effects for a layered surface, to a traditional RGB BRDF approximation of the local light reflection. This approach allows to compute the interferences between the light that is directly reflected and the part the light that have traversed the layer, but also to create color dispersion when the refraction index is non-uniform in the layer, and when the two boundaries of the layer are not parallel.

We have presented two implementations of this approach, a Phong-like specular reflection, and a diffuse model. Even if this models is not completely physically based, these implementations show that realistic effects can be achieved by adjusting a small set of intuitive parameters. This allows for computing a large range of surface appearances that are based on layered materials. This BRDF can also be implemented using the current graphic hardware facilities. 


\section{Future work}

Since this approach has not been design to be physically accurate, but only to create convincing effects, it has some drawbacks, as the lack of precision. However, it will be interesting to use this model to fit some measured BRDF data to it. Due to the low number of parameters, it will be easier to fit the measured data to this representation. A simple non-linear optimization approach should be sufficient.

Although this model show that wavelength dependent effects are possible by using only the RGB representation of color, a more accurate representation can be investigated. By using the projection function from a spectral representation of the light to the RGB component, a more physical model can be developed. The main problem is here to find the correct approximation to be able to find simple solutions to the resulting integration.

\section{Acknowledgments}

Special thanks to Lionel Bastard, for the productive discussions. He provides a lot of remarks and comments during the development of this model.

\section{References}

Ashikhmin, M., Premoze, S., Shirley, P., Jul. 2000. A microfacet-based brdf generator. In: Proc. of SIGGRAPH '00. ACM SIGGRAPH, ACM Press, pp. 65-74.

Blinn, J., August 1978. Simulation of wrinkled surfaces. In: Proc. of SIGGRAPH '78. Vol. 12. pp. 286-292.

Born, M., Wolf, E., 1975. Principles of Optics: Electromagnetic Theory of Propagation, Interference and Diffraction of Light. Pergamo Press, Oxford.

Hanrahan, P., Krueger, W., August 1993. Reflection from layered surfaces due to subsurface scattering. In: Kajiya, T. (Ed.), Computer Graphics (SIGGRAPH '93 Proc.). Vol. 27. pp. 165-174.

He, X. D., Torrance, K. E., Sillion, F. X., , Greenberg, D. P., Jul. 1991. A comprehensive physical model for light reflection. In: Sederberg, T. W. (Ed.), Computer Graphics (SIGGRAPH '91 Proc.). Vol. 25. pp. 175-186.

Hirayama, H., Kaneda, K., Yamashita, H., , Monden, Y., september 2000a. An accurate illumination model for objects coated with multilayer films. In: de Sousa, A., Torres, J. (Eds.), Eurographics 2000 Short Presentations. Eurographics, Eurographics, pp. 145-150. 
Hirayama, H., Kaneda, K., Yamashita, H., Yamaji, Y., Monden, Y., 1999. Visualization of optical phenomena caused by multilayer films with complex refractive indices. In: Proc. Pacific Graphics '99. pp. 128-137.

Hirayama, H., Kaneda, K., Yamashita, H., Y.Yamaji, Monden, Y., 2001. Visualization of optical phenomena caused by multilayer films based on wave optics. The Visual Computer 17, 106-120.

Hirayama, H., Yamajia, Y., adn Hideo Yamashita adn Yoshimi Monden, K. K., 2000b. Rendering iridescent colors appearing on a natural object. In: Proc. Pacific Graphics 2000. pp. 15-22.

Icart, I., Arques, D., June 2000. A physically-based brdf model for multilayer systems with uncorrelated rough boundaries. In: Bernard Peroche, H. R. (Ed.), Eurographics Rendering Workshop 2000. Eurographics, Eurographics, pp. 353-364.

Icart, I., Arqus, D., June 1999. An illumination model for a system of isotropic substrate-isotropic thin film with identical rough boundaries. In: 10th Eurographics Workshop on Rendering. Springer Computer Sciences, pp. 261-272.

Lafortune, E. P., Willems, Y. D., Novembre 1994. Using the modified phong reflectance model for physically based rendering. Tech. Rep. CW 197, Departement of Computing Science, K.U. Leuven.

Lafortune, E. P. F., Foo, S.-C., Torrance, K. E., Greenberg, D. P., August 1997. Non-linear approximation of reflectance function. In: Proc. of SIGGRAPH '97. ACM SIGGRAPH, ACM Press, pp. 117-126.

Perlin, K., Jul. 1985. An image synthesizer. In: Barsky, B. A. (Ed.), Computer Graphics (SIGGRAPH '85 Proc.). Vol. 19. pp. 287-296.

Phong, B.-T., 1975. Illumination for computer generated pictures. CACM June 197518 (6), 311-317.

Schlick, C., september 1994. An inexpensive brdf model for physically-based rendering. In: Proc. Eurographics'94. Vol. 13. Eurographics, Eurographics, pp. 233-246.

Stam, J., August 1999. Diffraction shader. In: Glassner, A. (Ed.), Proc. of SIGGRAPH '99. ACM SIGGRAPH, ACM Press, pp. 75-84.

Sun, Y., Fracchia, D., Drew, M., Calvert, T., June 2000. Rendering iridescent colors of optical disks. In: Bernard Peroche, H. R. (Ed.), Eurographics Rendering Workshop 2000. Eurographics, Eurographics, pp. 353-364.

Torrance, K. E., Sparrow, E. M., 1967. Theory for off-specular reflection from roughened surfaces. Journal of Optical Society of America 59 (9).

Ward, G. J., Jul. 1991. Measuring and modeling anisotropic reflection. In: Catmull, E. E. (Ed.), Computer Graphics (SIGGRAPH '92 Proc.). Vol. 26. pp. 265-272. 